\title{
Mutual Independence of Critical Temperature and Superfluid Density under Pressure in Optimally Electron-Doped Superconducting $\mathrm{LaFeAsO}_{1-x} \mathrm{~F}_{x}$
}

\author{
G. Prando, ${ }^{1, *}$ Th. Hartmann, ${ }^{2}$ W. Schottenhamel, ${ }^{1}$ Z. Guguchia, ${ }^{3}$ S. Sanna, ${ }^{4}$ F. Ahn, ${ }^{2}$ \\ I. Nekrasov, ${ }^{5}$ C. G. F. Blum, ${ }^{1}$ A. U. B. Wolter, ${ }^{1}$ S. Wurmehl, ${ }^{1,6}$ R. Khasanov, ${ }^{3}$ \\ I. Eremin, ${ }^{2,7}$ and B. Büchner ${ }^{1,6}$ \\ ${ }^{1}$ Leibniz-Institut für Festkörper- und Werkstoffforschung (IFW) Dresden, D-01171 Dresden, Germany \\ ${ }^{2}$ Institut für Theoretische Physik III, Ruhr-Universität Bochum, D-44801 Bochum, Germany \\ ${ }^{3}$ Laboratory for Muon Spin Spectroscopy, Paul Scherrer Institut, \\ CH-5232 Villigen PSI, Switzerland \\ ${ }^{4}$ Dipartimento di Fisica and Unità CNISM di Pavia, Università di Pavia, I-27100 Pavia, Italy \\ ${ }^{5}$ Institute for Electrophysics, Russian Academy of Sciences, Ural Branch, \\ Ekaterinburg 620016, Russian Federation \\ ${ }^{6}$ Institut für Festkörperphysik, Technische Universität Dresden, D-01062 Dresden, Germany \\ ${ }^{7}$ Kazan (Volga region) Federal University, 420008 Kazan, Russian Federation \\ (Received 28 January 2015; published 17 June 2015; publisher error corrected 19 June 2015)
}

\begin{abstract}
The superconducting properties of $\mathrm{LaFeAsO}_{1-x} \mathrm{~F}_{x}$ under conditions of optimal electron doping are investigated upon the application of external pressure up to $23 \mathrm{kbar}$. Measurements of muon-spin spectroscopy and dc magnetometry evidence a clear mutual independence between the critical temperature $T_{c}$ and the low-temperature saturation value for the ratio $n_{s} / m^{*}$ (superfluid density over effective band mass of Cooper pairs). Remarkably, a dramatic increase of $\sim 30 \%$ is reported for $n_{s} / m^{*}$ at the maximum pressure value while $T_{c}$ is substantially unaffected in the whole accessed experimental window. We argue and demonstrate that the explanation for the observed results must take the effect of nonmagnetic impurities on multiband superconductivity into account. In particular, the unique possibility to modify the ratio between intraband and interband scattering rates by acting on structural parameters while keeping the amount of chemical disorder constant is a striking result of our proposed model.
\end{abstract}

DOI: 10.1103/PhysRevLett.114.247004

PACS numbers: 74.70.Xa, 74.20.Pq, 74.62.Fj, 76.75.+i

Experimental evidence together with the currently accepted theoretical models ascribe the wealth of electronic ground states exhibited by $R \mathrm{FeAsO}_{1-x} \mathrm{~F}_{x}$ ( $R$ is a rare-earth ion), and more generally by all iron-based pnictides, to a complex and hard-to-disentangle interplay of chemical charge doping and multiorbital degrees of freedom [1-8], quenched disorder [9-15], and lattice strains [16-19]. In this respect, an interesting alternative to modify the behavior of pnictides in a more controlled way, i.e., by keeping the chemical levels of charge doping and quenched disorder constant, is to apply an external pressure $P$. $R \mathrm{FeAsO} \mathrm{1}_{1-x} \mathrm{~F}_{x}$ compounds have been shown indeed to be a fertile ground to investigate the effects of pressure across the whole electronic phase diagram [20-25]. Accordingly, pressure may be employed as a crucial tool in order to clarify important issues in these materials concerning, in particular, the exotic nature of the superconducting ground state. As a well-known result in the study of high- $T_{c}$ superconductivity (SC), a universal linear scaling is reported for the critical temperature $T_{c}$ vs $n_{s}(0) / m^{*}$, i.e., the ratio between the low-temperature $T$ saturation value of the superfluid density $n_{s}$ and the effective band mass $m^{*}$ of Cooper pairs. The so-called Uemura relation $T_{c} \propto$ $n_{s}(0) / m^{*}$ is obeyed indeed by several materials quantitatively, i.e., with the same slope, giving strong evidence for a breakdown of the weak-coupling scenario and, in turn, for unconventional SC [26-29]. The first investigations of $n_{s}$ in $R \mathrm{FeAsO} \mathrm{A}_{1-x} \mathrm{~F}_{x}$ seemed to reconcile it well to other superconductors [30,31]. However, more extensive reports on several samples of different origin show indeed that $R \mathrm{Fe} A s \mathrm{O}_{1-x} \mathrm{~F}_{x}$ superconductors do not obey the Uemura relation [32]. As these results may be critically influenced by the different conditions of doping and quenched disorder in the considered samples, clarifying measurements limiting the effect of chemical perturbations are needed for the aim of more consistent results.

In this Letter, we report on an investigation of the effect of $P \lesssim 23 \mathrm{kbar}$ in an optimally electron-doped sample of $\mathrm{LaFeAsO}_{1-x} \mathrm{~F}_{x}$. Our results of dc magnetometry confirm the absence of a positive effect of $P$ on $T_{c}$ [22]. At the same time, we point out by means of muon spin rotation $\left(\mu^{+} \mathrm{SR}\right)$ that $\mathrm{SC}$ is remarkably reinforced by pressure, as reflected in a dramatic enhancement of $n_{s}(0) / m^{*}$ by $\sim 30 \%$ at the maximum $P$ value. Such a dramatic increase under pressure while keeping $T_{c}$ constant is an unprecedented observation for any superconducting material, to the best of our knowledge. We provide evidence from density functional theory (DFT) calculations that this result should not be associated with an induced change in the fermiology of $\mathrm{LaFeAsO}_{1-x} \mathrm{~F}_{x}$ or, equivalently, to a pressure dependence 
of $m^{*}$. We argue that the observed effect can only emerge in a multiband superconductor in the presence of nonmagnetic impurities such as, e.g., As vacancies [33]. Strikingly, the mutual independence of $T_{c}$ and $n_{s}(0)$ can be explained within a multiband model by assuming that pressure modifies the ratio between intraband and interband impurity scattering rates by only acting on structural parameters while keeping the amount of chemical quenched disorder constant.

The impact of pressure on $n_{s}$ was investigated in a polycrystalline sample of optimally doped $\mathrm{LaFeAsO}_{1-x} \mathrm{~F}_{x}$ (with measured $x=0.13 \pm 0.01$ [34]) by means of transverse-field (TF) $\mu^{+}$SR performed on the General Purpose Decay-Channel (GPD) spectrometer, $\mu \mathrm{E} 1$ beam line, at the Paul Scherrer Institute, Switzerland. Figure 1 shows representative TF depolarization curves obtained for the sample loaded in the pressure cell at ambient pressure and for $T$ values well above and well below $T_{c} \simeq 24 \mathrm{~K}$ [Figs. 1(a) and 1(b), respectively]. The external transverse magnetic field $B_{\mathrm{ext}}=600 \mathrm{G}$ induces a coherent precession of implanted muons $\mu^{+}$(the condition $B_{c 1} \ll B_{\text {ext }} \ll B_{c 2}$ holds, $B_{c 1}$ and $B_{c 2}$ being the lower and upper critical fields for the superconductor, respectively). For $T \gg T_{c}$, this precession is only weakly damped by the electronic magnetism of the pressure cell material and by the nuclear moments of both sample and pressure cell material, while a much higher damping is observed for $T \ll T_{c}$. The details of the time-domain fitting of our experimental data [see the continuous lines in Figs. 1(a) and 1(b)] are described in the Supplemental Material [34]. Fast Fourier transforms (FFTs)

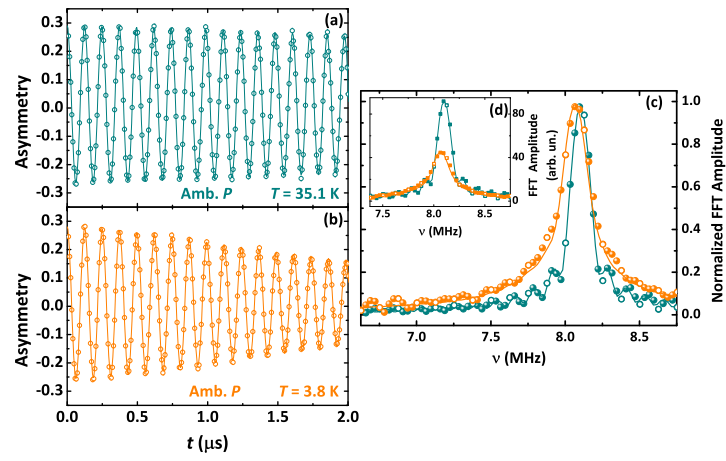

FIG. 1 (color online). Open points in (a) and (b) are experimental TF- $\mu^{+}$SR depolarization curves at representative $T$ values well above and well below $T_{c} \simeq 24 \mathrm{~K}$, respectively, (data are relative to ambient pressure and $B_{\text {ext }}=600 \mathrm{G}$ ), while the solid lines are time-domain fitting curves (see text). FFTs are performed with no preliminary apodization and the normalized spectra of the experimental data in (a) and (b) are reported in (c) as open symbols using the same color code [non-normalized FFT spectra are displayed in (d)]. The solid symbols in (c) and (d) are the results of FFTs after a preliminary zero padding extending up to 4 times the experimental window. The FFTs of the fitting curves in (a) and (b) are reported as solid lines in (c) and (d) after zero padding extending up to 4 times the experimental window. were performed with no preliminary data apodization in order to avoid artificial extra broadening. FFT spectra of both the experimental data and fitting curves are reported in Figs. 1(c) and 1(d), evidencing for $T \ll T_{c}$ both a shift of the signal to lower frequencies and the extra broadening discussed above. These are the well-known $\mu^{+}$SR signatures of type-II superconductivity. The former effect is due to the diamagnetism in the superconducting phase, while the latter is due to the modulation of $B_{\mu}$, namely, the local magnetic field at the $\mu^{+}$site, introduced by the vortex glass phase in the mixed Shubnikov state of the $H-T$ phase diagram. The detailed properties of this modulation are determined by the penetration depth $\lambda$, whose temperature dependence can be derived accordingly. Asymmetric FFT spectra are typically expected for $T \ll T_{c}$ in type-II superconductors $[44,45]$. However, this feature is hindered in the current measurements by the polycrystalline nature of the sample and by the strong influence of the stray magnetic fields from the superconducting phase on the pressure cell [46]. Accordingly, the induced extra broadening $\sigma_{\mathrm{sc}}$ is of Gaussian character and one has indeed $\sigma_{\mathrm{sc}}(T) \propto \lambda(T)^{-2} \propto$ $n_{s}(T) / m^{*}$ [32,44-48].

Results of $\sigma_{\mathrm{sc}}$ as a function of both $T$ and $P$ for the current $\mathrm{LaFeAsO}_{1-x} \mathrm{~F}_{x}$ sample are presented in Fig. 2. $\sigma_{\mathrm{sc}}(T)$ can be tracked fairly well by a single-gap $s$-wave behavior as shown by the solid lines in Fig. 2, in good agreement with previous $\mu^{+} \mathrm{SR}$ reports on similar samples [30]. The actual $\sigma_{\mathrm{sc}}(T)$ dependence will not be considered any longer in this Letter and its discussion will be postponed to another work. A sizeable enhancement $(\sim 30 \%)$ of the saturation value $\sigma_{\mathrm{sc}}(0)$ is clearly noticed upon increasing $P$. However, it must be stressed that, as displayed in the inset of Fig. 2 for data normalized over the respective $\sigma_{\mathrm{sc}}(0)$ values, no qualitative change is observed for the actual $\sigma_{\mathrm{sc}}(T)$ shape at different $P$ values. At the same time, the data in Fig. 2 remarkably show that $T_{c}$ is unaffected by $P$. This latter observation is independently

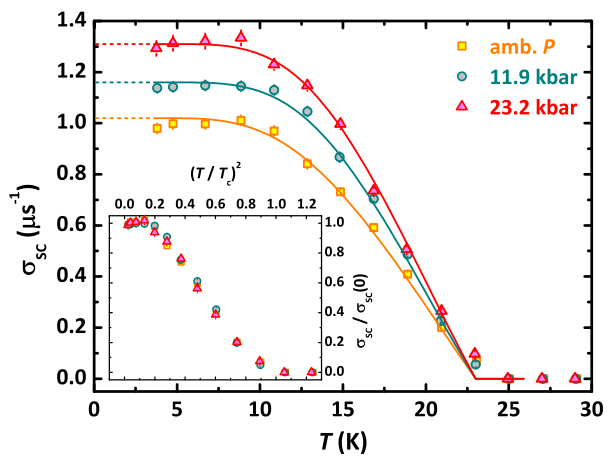

FIG. 2 (color online). Main panel: $\sigma_{\mathrm{sc}}$ as a function of $T$ at the different investigated $P$ values. Solid lines are best-fit curves according to a single-gap $s$-wave behavior, whose extrapolations to the $T=0$ limit are shown by dashed lines. Data are also reported in the inset using the same symbols as a function of $\left(T / T_{c}\right)^{2}$ and after normalization over the respective $\sigma_{\mathrm{sc}}(0)$ values. 
confirmed by means of dc magnetometry, as shown in Fig. 3 (details in the Supplemental Material [34]). Measurements of the dc magnetization $(M)$ were performed at $H=10$ Oe under zero-field cooling (ZFC) conditions and the results clearly evidence that the superconducting transition of the sample is only weakly modified by pressure. At most, $T_{c}$ is even slightly shifted to lower values at the maximum $P$ value, as shown in the inset of Fig. 3. The shielding fraction is also not affected at all by $P$ and it is $\sim 100 \%$, as displayed by the calibration measurement performed without the pressure cell (full symbols in Fig. 3, main panel). The trend observed for our results is in excellent agreement with what is reported in the literature for a nominally identical compound [22]. Misleading reports on the effect of pressure on $\mathrm{SC}$ in $R \mathrm{FeAsO}{ }_{1-x} \mathrm{~F}_{x}$ often make use of the broadened resistive SC transition to claim a dramatic increase of $T_{c}$ upon increasing $P$ [49-51]. However, it should be remarked that bulk magnetic estimates of the real thermodynamic $T_{c}$ point out a much more modest effect of $P$ and, interestingly, the $T_{c}$ values upon increasing $P$ seem to be limited by the value obtained at the optimal chemical doping [22].

Our experimental findings unambiguously show that $T_{c}$ and $\sigma_{\mathrm{sc}}(0)$ are uncorrelated quantities upon increasing $P$, clearly implying a breakdown of the Uemura relation in $\mathrm{LaFeAsO}_{1-x} \mathrm{~F}_{x}$. In the case of cuprates, $T_{c} \propto n_{s}(0) / m^{*}$ has been confirmed in the underdoped region of the electronic phase diagram [26-29]. However, clear deviations have been observed in the past, probably the most remarkable one being reported for $\mathrm{Y}_{1-x} \mathrm{Ca}_{x} \mathrm{Ba}_{2} \mathrm{Cu}_{3} \mathrm{O}_{7-\delta}$ as a function of chemical substitutions. Here, a clear saturation effect for $T_{c}$ with $n_{s}$ strongly increasing was observed and explained in terms of different contributions to $n_{s}$ arising from carriers

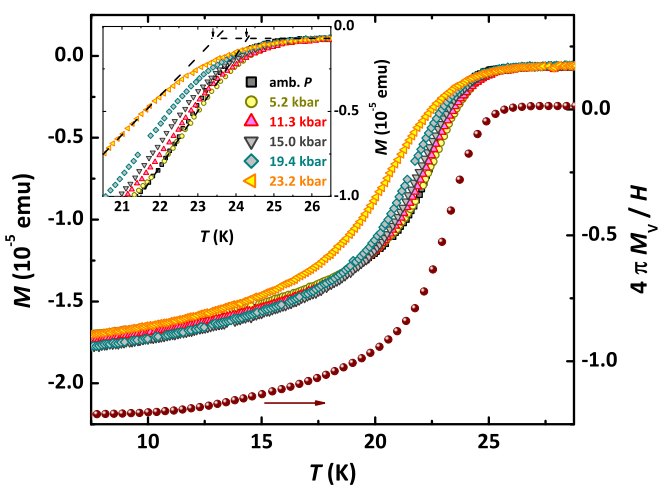

FIG. 3 (color online). Main panel: dc magnetization $M$ as a function of $T$ for different $P$ values (open symbols, left scale). Measurements were performed under ZFC conditions $(H=10 \mathrm{Oe})$ and a reference curve was taken under ZFC conditions $(H=5 \mathrm{Oe})$ with the sample without the pressure cell (solid symbols, right scale, in $1 / 4 \pi$ units). An enlargement of the data in the region of the superconducting transition is displayed in the inset, where the dashed lines define $T_{c}$ values, indicated by arrows. on $\mathrm{CuO}_{2}$ planes and $\mathrm{CuO}$ chains [52]. A similar effect was measured in $\mathrm{YBa}_{2} \mathrm{Cu}_{3} \mathrm{O}_{7-\delta}$ upon increasing $P$ [46]. However, the relative enhancement of $n_{s}$ under pressure is $\sim 3$ times smaller than what is observed in the current case for $\mathrm{LaFeAsO}_{1-x} \mathrm{~F}_{x}$ and, at the same time, the explanation can still rely on charge contributions from planes and chains to $n_{s}$, an argument that is not suited at all to $\mathrm{LaFeAsO}_{1-x} \mathrm{~F}_{x}$. These issues denote deep intrinsic distinctions indicative of a different underlying physics and, accordingly, novel scenarios should be considered to correctly understand our observations.

As discussed above, the measured extra broadening $\sigma_{\mathrm{sc}}$ of the $\mu^{+}$SR spectrum mainly depends on two contributions, namely, $n_{s}$ and $m^{*}$. However, a strong pressure dependence of $m^{*}$ can be ruled out by DFT calculations of the electronic band structure. In particular, as shown in Fig. 4, electronic bands (and their inverse second derivatives) computed in the local density approximation within the linearized muffin-tin orbitals method using default settings are only weakly affected by pressure even up to 260 kbar, namely, much beyond the experimentally accessible range. This is in agreement with previous reports for lower $P$ values [20]. Including low-energy electronic correlations beyond the local density approximation will certainly introduce a moderate renormalization of $m^{*}$, yet these correlations will typically also affect $T_{c}$ at the same time due to the modification of the low-energy part of the bosonic spectrum. Accordingly, to understand the experimental results on a quantitative level, we focus directly on the intrinsic density of supercarriers $n_{s}$. We start from a model for the $s^{ \pm}$superconductor that consists of superconducting gaps of similar magnitudes but of opposite signs formed on hole $(h)$ and electron $(e)$ bands $[1,2,53,54]$. The electron pockets are located around the $X=(\pi, 0)$ points of the Brillouin zone, which have lower symmetry than the $\Gamma=(0,0)$ or the $M=(\pi, \pi)$ points, where hole pockets are located. Accordingly, we allow for a $\cos 2 \theta$ modulation of the gap on the electron pocket [6]

$$
\Delta_{e}(\theta)=\Delta_{e} \pm \Delta_{\bar{e}} \cos 2 \theta \quad\left(\text { where } \Delta_{e}=-\Delta_{h}\right) .
$$

A modification of the mediating boson responsible for SC under pressure, i.e., pressure-dependent intraband $\left(\lambda_{e e}\right.$ and $\left.\lambda_{h h}\right)$ and interband $\left(\lambda_{h e}\right)$ couplings, may well affect $n_{s}(0)$. However, the modification of $\lambda$ couplings alone is not

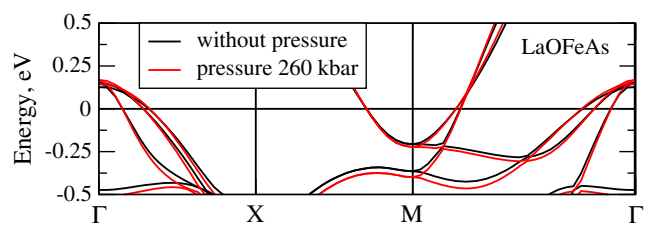

FIG. 4 (color online). Band structure of $\mathrm{LaFeAsO}$ at ambient pressure (black curves) and for $P=260 \mathrm{kbar}$ (red curves). 
enough to justify our results, as this would simultaneously modify $T_{c}$ as well.

Following the discussion above, we argue that a mutual independence of $n_{s}(0)$ and $T_{c}$ with increasing pressure may only be obtained by taking the effect of scattering from nonmagnetic impurities into account. Because of the internal sign change of the gap between the $h$ and $e$ pockets, the effect of such impurities can be separated into two contributions. The intraband scattering rate $\Gamma_{0}$ within $h$ and/or $e$ pockets does not affect the superconducting gap and $T_{c}$ for $s$-wave symmetry [55-58]. On the other hand, the interband scattering rate $\Gamma_{\pi}$ between $h$ and $e$ pockets leads to strong pair-breaking effects, similarly to the case of magnetic scattering $[9,11,59]$. In particular, while a change of $\Gamma_{0}$ only affects the superfluid density and keeps $T_{c}$ constant, $\Gamma_{\pi}$ affects both quantities in a similar fashion [9]. Nonmagnetic impurities change the balance between the angle-independent and angle-dependent parts of the gap on the $e$ pockets as well. In the presence of impurities, one identifies the new Matsubara frequencies $i \tilde{\omega}_{m}^{h}=i \omega_{m} \eta_{m}^{h}$ and $i \tilde{\omega}_{m}^{e}=i \omega_{m} \eta_{m}^{e}$ for $h$ and $e$ pockets, respectively, as well as the superconducting gaps $\tilde{\Delta}_{m}^{h}=\bar{\Delta}_{m}^{h} \eta_{m}^{h}$ and $\tilde{\Delta}_{m}^{e}(\theta)=\bar{\Delta}_{m}^{e}(\theta) \eta_{m}^{e}$. All of these quantities are determined self-consistently for a given temperature and impurity scattering as described in the Supplemental Material [34]. Finally, the expression for $n_{s}(T)$, i.e., the zerofrequency value of the current-current correlation function, can be written as

$$
\begin{aligned}
\frac{n_{s}(T)}{n_{s}(0)}= & \frac{\pi T}{2} \sum_{\omega_{m}}\left[\frac{\left|\bar{\Delta}_{m}^{h}\right|^{2}}{\eta_{m}^{h}\left(\omega_{m}^{2}+\left|\bar{\Delta}_{m}^{h}\right|^{2}\right)^{3 / 2}}\right. \\
& \left.+\left\langle\frac{\left|\bar{\Delta}_{m}^{e}\right|^{2}}{\eta_{m}^{e}\left(\omega_{m}^{2}+\left|\bar{\Delta}_{m}^{e}(\theta)\right|^{2}\right)^{3 / 2}}\right\rangle_{\theta}\right] .
\end{aligned}
$$

We now assume that the multiband nature of SC in pnictides allows pressure to modify the $\Gamma_{\pi} / \Gamma_{0}$ ratio while still keeping the chemical density of nonmagnetic impurities constant. The striking result of our calculations is that a relatively small variation of $\Gamma_{\pi} / \Gamma_{0}$ is indeed able to reproduce well the experimental data, as shown in Fig. 5, where a clear decoupling of $n_{s}$ and $T_{c}$ is reported. It must be noticed that such a behavior would be characteristic for any multiband superconductor independently on the phase structure of the superconducting gap on the different Fermi surfaces, namely for both $s^{ \pm}$and $s^{++}$. It should be pointed out as well that significant changes of $\Gamma_{\pi}$ with increasing pressure are ruled out, as they would affect the actual $T$ dependence of $n_{s}$ [9], opposite to the experimental findings reported in Fig. 2. Finally, it is worth stressing that assuming a modification in $\Gamma_{\pi} / \Gamma_{0}$ implicitly involves a pressure dependence of the orbital composition of the $t_{2 g}$ states at the Fermi energy and, accordingly, an important orbital character of superconductivity. While these arguments have already been discussed on a more quantitative
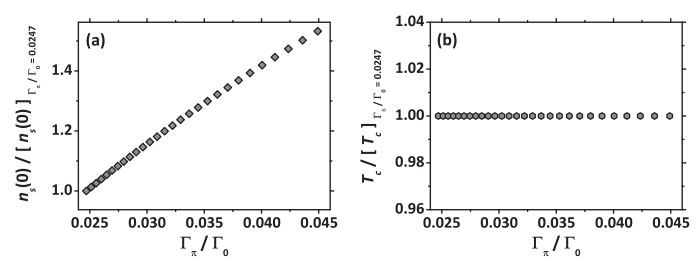

FIG. 5. Calculated behavior of $n_{s}(0)$ and $T_{c}[(\mathrm{a})$ and (b), respectively] as a function of the $\Gamma_{\pi} / \Gamma_{0}$ ratio. Both quantities are normalized to their values at $\Gamma_{\pi} / \Gamma_{0}=0.0247$. We further employ $m_{h}=2.0 m$ and $m_{e}=1.5 m$ with energy dispersions $\xi_{h}=-k^{2} /\left(2 m_{h}\right)+\mu \quad$ and $\quad \xi_{e}=k^{2} /\left(2 m_{e}\right)-\mu-\mu_{0}$. Here, $\mu=3$ and $\mu_{0}=0.2$.

basis for other pnictide superconductors at ambient pressure [6], nothing is directly known for $R \mathrm{Fe} \mathrm{AsO}_{1-x} \mathrm{~F}_{x}$ due to the current lack of angle-resolved photoemission spectroscopy (ARPES) data. As such, our results give an interesting hint towards a universal behavior shared by different families of pnictides.

Unfortunately, usual experimentally accessible quantities (like the residual resistance ratio or the mean free path) mostly involve the overall effects of impurities (i.e., $\Gamma=\Gamma_{0}+\Gamma_{\pi}$ ) rather than the $\Gamma_{\pi} / \Gamma_{0}$ ratio, which is relevant for our current analysis. Nevertheless, certain quantitative predictions can still be made out of our model. In particular, our calculations show that an increase of $n_{s}$ occurs either when $\Gamma$ stays constant or slightly decreases upon increasing $P$. Moreover, we also find that $\Gamma_{0} \gg \Gamma_{\pi}$, consistent with our experimental findings.

Summarizing, we have reported on the superconducting properties of optimally electron-doped $\mathrm{LaFeAsO}_{1-x} \mathrm{~F}_{x}$ under pressure. A breakdown of the Uemura relation is unambiguously evidenced, with $T_{c}$ unaffected by pressure and an increase of $n_{s}(0) / \mathrm{m}^{*}$ by $\sim 30 \%$ at $P \simeq 23 \mathrm{kbar}$. We propose an explanation based on the unique possibility to modify the ratio between intraband and interband scattering rates in multiband superconductors by only acting on structural parameters while keeping the amount of chemical disorder constant. This scenario can be accounted for by assuming an important orbital character of superconductivity in $\mathrm{LaFeAsO}_{1-x} \mathrm{~F}_{x}$.

We acknowledge useful and stimulating discussions with A. V. Chubukov, S.-L. Drechsler, D. V. Efremov, and G. A. Ummarino. G. P. acknowledges support by the Humboldt Research Fellowship for Postdoctoral Researchers. The work of Th.H., F. A., S. W., I.,E., and B. B. is supported by the Deutsche Forschungsgemeinschaft under the Priority Program SPP 1458. I.E, T. H., and F. A. acknowledge support by the German Academic Exchange Service (DAAD PPP USA No. 57051534). S. S. acknowledges partial support of PRIN2012 Project No. 2012X3YFZ2. I. N. acknowledges support by RFBR Grant No. 14-02-00065. S. W. acknowledges funding by the Deutsche Forschungsgemeinschaft in the Emmy 
Noether project WU595/3-2. I. E. thanks the Platform for Superconductivity and Magnetism Dresden for its kind hospitality. The work of I. E. was supported by the Russian Federation Government Program of Competitive Growth of Kazan (Volga region). We thank R. Wachtel, S. MüllerLitvanyi, and G. Kreutzer for technical support.

*g.prando@ifw-dresden.de

[1] A. V. Chubukov, D. V. Efremov, and I. Eremin, Phys. Rev. B 78, 134512 (2008).

[2] I. I. Mazin and J. Schmalian, Phys. C 469, 614 (2009).

[3] S. Maiti, M. M. Korshunov, T. A. Maier, P. J. Hirschfeld, and A. V. Chubukov, Phys. Rev. Lett. 107, 147002 (2011).

[4] A. V. Chubukov, Annu. Rev. Condens. Matter Phys. 3, 57 (2012).

[5] R. M. Fernandes, A. V. Chubukov, and J. Schmalian, Nat. Phys. 10, 97 (2014).

[6] R. Yu, J.-X. Zhu, and Q. Si, Phys. Rev. B 89, 024509 (2014)

[7] S.-H. Baek, D. V. Efremov, J. M. Ok, J. S. Kim, J. van den Brink, and B. Büchner, Nat. Mater. 14, 210 (2014).

[8] I. Eremin, J. Knolle, R. M. Fernandes, J. Schmalian, and A. V. Chubukov, J. Phys. Soc. Jpn. 83, 061015 (2014).

[9] A. B. Vorontsov, M. G. Vavilov, and A. V. Chubukov, Phys. Rev. B 79, 140507 (2009).

[10] D. V. Efremov, M. M. Korshunov, O. V. Dolgov, A. A. Golubov, and P. J. Hirschfeld, Phys. Rev. B 84, 180512 (2011).

[11] M. G. Vavilov and A. V. Chubukov, Phys. Rev. B 84, 214521 (2011).

[12] R. M. Fernandes, M. G. Vavilov, and A. V. Chubukov, Phys. Rev. B 85, 140512 (2012).

[13] Y. Bang, H.-Y. Choi, and H. Won, Phys. Rev. B 79, 054529 (2009).

[14] S. Onari and H. Kontani, Phys. Rev. Lett. 103, 177001 (2009).

[15] G. Prando, O. Vakaliuk, S. Sanna, G. Lamura, T. Shiroka, P. Bonfà, P. Carretta, R. De Renzi, H.-H. Klauss, C. G. F. Blum, S. Wurmehl, C. Hess, and B. Büchner, Phys. Rev. B 87, 174519 (2013).

[16] G. Garbarino, P. Toulemonde, M. Álvarez-Murga, A. Sow, M. Mezouar, and M. Núñez-Regueiro, Phys. Rev. B 78, 100507 (2008).

[17] K. Kuroki, H. Usui, S. Onari, R. Arita, and H. Aoki, Phys. Rev. B 79, 224511 (2009).

[18] G. Garbarino, R. Weht, A. Sow, A. Sulpice, P. Toulemonde, M. Álvarez-Murga, P. Strobel, P. Bouvier, M. Mezouar, and M. Núñez-Regueiro, Phys. Rev. B 84, 024510 (2011).

[19] J. Kang, A. F. Kemper, and R. M. Fernandes, Phys. Rev. Lett. 113, 217001 (2014).

[20] R. De Renzi, P. Bonfà, M. Mazzani, S. Sanna, G. Prando, P. Carretta, R. Khasanov, A. Amato, H. Luetkens, M. Bendele, F. Bernardini, S. Massidda, A. Palenzona, M. Tropeano, and M. Vignolo, Supercond. Sci. Technol. 25, 084009 (2012).

[21] R. Khasanov, S. Sanna, G. Prando, Z. Shermadini, M. Bendele, A. Amato, P. Carretta, R. De Renzi, J. Karpinski, S. Katrych, H. Luetkens, and N. D. Zhigadlo, Phys. Rev. B 84, 100501(R) (2011).
[22] K. Miyoshi, E. Kojima, S. Ogawa, Y. Shimojo, and J. Takeuchi, Phys. Rev. B 87, 235111 (2013).

[23] G. Prando, P. Bonfà, G. Profeta, R. Khasanov, F. Bernardini, M. Mazzani, E. M. Brüning, A. Pal, V. P. S. Awana, H.-J. Grafe, B. Büchner, R. De Renzi, P. Carretta, and S. Sanna, Phys. Rev. B 87, 064401 (2013).

[24] G. Prando, S. Sanna, R. Khasanov, A. Pal, E. M. Brüning, M. Mazzani, V. P. S. Awana, B. Büchner, and R. De Renzi, J. Phys. Chem. Solids Suppl. 84, 63 (2015).

[25] C. A. McElroy, J. J. Hamlin, B. D. White, S. T. Weir, Y. K. Vohra, and M. B. Maple, Phys. Rev. B 90, 125134 (2014).

[26] Y. J. Uemura, V. J. Emery, A. R. Moodenbaugh, M. Suenaga, D. C. Johnston, A. J. Jacobson, J. T. Lewandowski, J. H. Brewer, R. F. Kiefl, S. R. Kreitzman, G. M. Luke, T. Riseman, C. E. Stronach, W. J. Kossler, J. R. Kempton, X. H. Yu, D. Opie, and H. E. Schone, Phys. Rev. B 38, 909 (1988).

[27] Y. J. Uemura et al., Phys. Rev. Lett. 62, 2317 (1989).

[28] Y. J. Uemura, L. P. Le, G. M. Luke, B. J. Sternlieb, W. D. Wu, J. H. Brewer, T. M. Riseman, C. L. Seaman, M. B. Maple, M. Ishikawa, D. G. Hinks, J. D. Jorgensen, G. Saito, and H. Yamochi, Phys. Rev. Lett. 66, 2665 (1991).

[29] Y. J. Uemura, J. Phys. Condens. Matter 16, S4515 (2004).

[30] H. Luetkens, H.-H. Klauss, R. Khasanov, A. Amato, R. Klingeler, I. Hellmann, N. Leps, A. Kondrat, C. Hess, A. Köhler, G. Behr, J. Werner, and B. Büchner, Phys. Rev. Lett. 101, 097009 (2008).

[31] J. P. Carlo, Y. J. Uemura, T. Goko, G. J. MacDougall, J. A. Rodriguez, W. Yu, G. M. Luke, Pengcheng Dai, N. Shannon, S. Miyasaka, S. Suzuki, S. Tajima, G. F. Chen, W. Z. Hu, J. L. Luo, and N. L. Wang, Phys. Rev. Lett. 102, 087001 (2009).

[32] P. Carretta, R. De Renzi, G. Prando, and S. Sanna, Phys. Scr. 88, 068504 (2013).

[33] F. Hammerath, S.-L. Drechsler, H.-J. Grafe, G. Lang, G. Fuchs, G. Behr, I. Eremin, M. M. Korshunov, and B. Büchner, Phys. Rev. B 81, 140504 (2010).

[34] See Supplemental Material at http://link.aps.org/ supplemental/10.1103/PhysRevLett.114.247004, which includes Refs. [33-41], for detailed information about the characterization of the sample, both structural and compositional. The magnetic characterization of the sample by means of ambient-pressure $\mu^{+} \mathrm{SR}$ is provided as well. Finally, technical details about pressure measurements ( $\mu^{+} \mathrm{SR}$, dc magnetometry) and about the theoretical framework are given.

[35] A. Kondrat, J. E. Hamann-Borrero, N. Leps, M. Kosmala, O. Schumann, A. Köhler, J. Werner, G. Behr, M. Braden, R. Klingeler, B. Büchner, and C. Hess, Eur. Phys. J. B 70, 461 (2009).

[36] Y. Kamihara, T. Watanabe, M. Hirano, and H. Hosono, J. Am. Chem. Soc. 130, 3296 (2008).

[37] H. Luetkens, H.-H. Klauss, M. Kraken, F. J. Litterst, T. Dellmann, R. Klingeler, C. Hess, R. Khasanov, A. Amato, C. Baines, M. Kosmala, O. J. Schumann, M. Braden, J. Hamann-Borrero, N. Leps, A. Kondrat, G. Behr, J. Werner, B. Büchner, Nat. Mater. 8, 305 (2009).

[38] A. Yaouanc and P. Dalmas de Réotier, Muon Spin Rotation, Relaxation, and Resonance: Applications to Condensed Matter (Oxford University Press, Oxford, 2011). 
[39] D. R. Harshman, G. Aeppli, E. J. Ansaldo, B. Batlogg, J. H. Brewer, J. F. Carolan, R. J. Cava, M. Celio, A. C. D. Chaklader, W. N. Hardy, S. R. Kreitzman, G. M. Luke, D. R. Noakes, and M. Senba, Phys. Rev. B 36, 2386 (1987).

[40] J. E. Sonier, R. F. Kiefl, J. H. Brewer, D. A. Bonn, J. F. Carolan, K. H. Chow, P. Dosanjh, W. N. Hardy, R. Liang, W. A. MacFarlane, P. Mendels, G. D. Morris, T. M. Riseman, and J. W. Schneider, Phys. Rev. Lett. 72, 744 (1994).

[41] K. Yokogawa, K. Murata, H. Yoshino, and S. Aoyama, Jpn. J. Appl. Phys. 46, 3636 (2007).

[42] P. L. Alireza and G. G. Lonzarich, Rev. Sci. Instrum. 80, 023906 (2009).

[43] Y. Bang and G. R. Stewart, arXiv:1410.1244v2.

[44] S. J. Blundell, Contemp. Phys. 40, 175 (1999).

[45] J. E. Sonier, J. H. Brewer, and R. F. Kiefl, Rev. Mod. Phys. 72, 769 (2000).

[46] A. Maisuradze, A. Shengelaya, A. Amato, E. Pomjakushina, and H. Keller, Phys. Rev. B 84, 184523 (2011).

[47] E. H. Brandt, Phys. Rev. B 37, 2349 (1988).

[48] B. Pümpin, H. Keller, W. Kündig, W. Odermatt, I. M. Savic, J. W. Schneider, H. Simmler, P. Zimmermann, E. Kaldis, S. Rusiecki, Y. Maeno, and C. Rossel, Phys. Rev. B 42, 8019 (1990).
[49] H. Takahashi, K. Igawa, K. Arii, Y. Kamihara, M. Hirano, and H. Hosono, Nature (London) 453, 376 (2008).

[50] H. Takahashi, H. Okada, K. Igawa, K. Arii, Y. Kamihara, S. Matsuishi, M. Hirano, H. Hosono, K. Matsubayashi, and Y. Uwatoko, J. Phys. Soc. Jpn. 77 Suppl. C, 78 (2008).

[51] H. Takahashi, H. Soeda, M. Nukii, C. Kawashima, T. Nakanishi, S. Iimura, Y. Muraba, S. Matsuishi, and H. Hosono, Sci. Rep. 5, 7829 (2015).

[52] J. L. Tallon, C. Bernhard, U. Binninger, A. Hofer, G. V. M. Williams, E. J. Ansaldo, J. I. Budnick, and C. Niedermayer, Phys. Rev. Lett. 74, 1008 (1995).

[53] G. A. Ummarino, M. Tortello, D. Daghero, and R. S. Gonnelli, Phys. Rev. B 80, 172503 (2009).

[54] G. A. Ummarino, Phys. Rev. B 83, 092508 (2011).

[55] A. A. Abrikosov and L. P. Gor'kov, Sov. Phys. JETP 8, 1090 (1959).

[56] A. A. Abrikosov and L. P. Gor'kov, Sov. Phys. JETP 9, 220 (1959).

[57] P. W. Anderson, J. Phys. Chem. Solids 11, 26 (1959).

[58] A. A. Abrikosov and L. P. Gor'kov, Phys. Rev. B 49, 12337 (1994).

[59] A. A. Golubov and I. I. Mazin, Phys. Rev. B 55, 15146 (1997). 